\title{
Ultrashort Peptide Theranostic Nanoparticles by Microfluidic-Assisted Rapid Solvent Exchange
}

\author{
Ming Ni, Guillaume Tresset, Ciprian Iliescu and Charlotte A. E. Hauser
}

\begin{abstract}
Ultrashort peptides (USPs), composed of three to seven amino acids, can self-assemble into nanofibers in pure water. Here, using hydrodynamic focusing and a solvent exchange method on a microfluidic setup, we convert these nanofibers into globular nanoparticles with excellent dimensional control and polydispersity. Thanks to USP nanocarriers' structure, different drugs can be loaded. We used Curcumin as a model drug to evaluate the performance of USP nanocarriers as a novel drug delivery vehicle. These nanoparticles can efficiently cross the cell membrane and possess nonlinear optical properties. Therefore, we envisage USP nanoparticles as promising future theranostic nanocarriers.
\end{abstract}

Index Terms - self-assembling peptides, theranostic nanoparticles, microfluidics.

\section{INTRODUCTION}

$\mathrm{P}$ eptides are intriguing building blocks for many types of nanostructures, varying from nanofibers to nanorods, nanoribbons, and many more.[1] Therefore, peptidebased nanomaterials have been widely used as tissue engineering scaffolds, [2] biosensors,[3-5] drug delivery vehicles,[6] and bioimaging probes.[7] Peptides can be a viable drug delivery vehicle alternative to lipid or polymeric micelles vesicles, [8,9] due to their unique characteristic of recognition by biological systems (e.g. Arg-Gly-Asp(RGD) is a well-known integrin-binding ligand for cell adhesion).[10] Ultrashort peptides (USPs) can be synthesized by solid phase or batch synthesis methods.[11] The ability of USPs to overcome the plasma membrane barrier of mammalian cells make them promising candidates as nanocarriers for drug delivery applications.[12]

The classical way of peptide nanoparticles preparation is through bulk mixing.[13] However, using bulk mixing method, due to the kinetics of self-assembly process, the size and polydispersity of nanocarriers cannot be controlled precisely. This aspect subsequently results in a lack of efficiency for drug delivery. Recent developments in microfluidics enabled us to synthesize nanostructured

The correspondence should be addressed to Ciprian Iliescu (ciprian.iliescu@imt.ro) and Charlotte Hauser (charlotte.hauser@kaust.edu.sa).

M. Mi is with GenScript, Zhenjiang, Jiangsu Province, P. R. China. (e-mail: ming1.ni@genescript.com)

G. Tresset is with Université Paris-Saclay, CNRS, Laboratoire de Physique des Solides, 91405 Orsay, France (e-mail : guillaume.tresset@universite-parissaclay.fr)

C. Iliescu is with National Institute for Research and Development in materials with excellent control of size, composition and morphology.[14] Different other nanomaterials have been generated successfully via microfluidic mixing methods, e.g. chitosan,[15] lipids,[16-18] and poly(lactic-coglycolic acid) based nanoparticles,[19-21] DNA-based nanoparticles,[22, 23] theranostic lipoplexes,[24] polysulfone, $[25,26]$ or multiple layer assembly for the synthesis of polymer microcapsules.[27] Reviews on engineering nanoparticles in microfluidic devices for drug delivery applications are available [28-32].

USPs are a novel class of rationally designed peptides that self-assemble to fibrous nanomaterials.[33] The peptides are composed of three to seven amino acids, with the advantage of an easy manufacturing process in large quantities at relatively low costs due their small size. USPs are amphiphilic in nature, consisting of an aliphatic amino acid tail of decreasing hydrophobicity and a hydrophilic head. These peptides self-assemble most likely via parallel-antiparallel $\alpha$-helical pair formation and subsequent stacking into fibers that reorganize to $\beta$-turn fibrils which condense to helical fiber bundles (33). These fibers are a few nanometer in diameter and at least micrometers but even up to centimeters in length that can further aggregate and form nanofibrous scaffolds in the form of macroscopic hydrogels.[33] USP hydrogels have been used successfully as 3D printing bioinks, [34, 35] wound dressings,[36, 37] tissue engineering[38] and in cancer therapy.[39, 40] These USPs can also be used as optical imaging probes, being visualized by secondharmonic microscopy.[41]

Herein, we report the preparation of self-assembled USP nanoparticles, using a uniform solvent exchange process enhanced by the microfluidics-based hydrodynamic flow focusing method. USP nanoparticles (USPN) were characterized using scanning electron microscopy (SEM) and dynamic light scattering (DLS) techniques. These nanoparticles were further used for curcumin (as a model drug) encapsulation to test their utility as nanocarriers for drug delivery applications. Due to the optical properties previously reported [41], we propose USPNs as a special class of theranostic nanocarriers.

Microtechnologies, IMT-Bucharest, Bucharest 077190, Romania and Academy of Romanian Scientists, Bucharest 050094, Romania (e-mail: ciprian.iliescu@imt.ro).

C. A. E. Hauser is with King Abdullah University of Science \& Technology, Division of Biological \& Environmental Science and Engineering, Laboratory for Nanomedicine, Thuwal 23955-6900, Kingdom of Saudi Arabia (e-mail: charlotte.hauser@kaust.edu.sa). 


\section{MAterials AND MethodS}

\section{A. Peptides}

Ultrashort peptides, Ac-LIVAGD-NH ${ }_{2}\left(\mathrm{LD}_{6}\right)$, Ac-LIVAGK$\mathrm{NH}_{2}\left(\mathrm{LK}_{6}\right)$, Ac-LIVAGK-C2-Biotin (LK 6 -Biotin), Ac-IVK$\mathrm{NH}_{2} \quad\left(\mathrm{IK}_{3}\right)$ were purchased from the American Peptide Company (Sunnyvale, CA, USA) with high purity ( $\geq 95 \%)$. Net peptide content varied between $60 \%$ and $90 \%$. Following reagents were purchased from the respective companies: Methanol (JTBaker), ethanol (Fisher) and 1, 1, 1, 3, 3, 3hexafluoro-2-propanol (HFIP, Merck).

\section{B. Device Fabrication}

The microfluidic device consists of two inlets: A central one for the aequeous peptide solution and a second one for different solvents. Figure 1a shows a detailed layout of the microfluidic setup. The central peptide stream was hydrodynamically focused between two side streams containing solvents. The dimensions of the focusing channel were $60 \mu \mathrm{m} \times 40 \mu \mathrm{m} \times 15 \mathrm{~mm}$. In order to vary the degree of focusing, the flow rate ratio can be easily adjusted by a pressurized pumping system (Fluigent, France) that incorporates pressurized reservoirs and mass flow controllers in a software controlled closed loop.

A detailed fabrication process of the microfluidic device can be found in [42]. Briefly, the structure of the microfluidic device was "printed" on a $300 \mu \mathrm{m}$-thick silicon wafer using two classical microfabrication processes: photolithography and deep RIE etching. The microfluidic channels were defined from top side of the wafer, while the etch-through holes were performed from the opposite side. $\mathrm{The}^{\mathrm{SiO}_{2}}$ layer was grown (thermal dry oxidation) from the surface of the silicon structure in order to achieve a full hydrophilic surface of the microfluidic channel. The microfluidic channel was closed using a Pyrex glass wafer by anodic bonding. After dicing, Nanoport microfluidic connectors were mounted on the Si surface of the device. An image with the fabricated microfluidic device is presented in Figure 1b. Besides the advantage of the robustness, reusability and good dimensional control of the microfluidic structure, the main advantage of the glass-silicon device is that it allows an "on-site" cleaning of the microfluidic channels.[43] In our case this cleaning was done by using the same procedure, but just filling the reservoirs with N-methyl pyrrolidone (NMP) solution letting it flow through the microfluidic setup, followed by cleaning with isopropyl alcohol (IPA) and deionized (DI) water.

\section{Preparation of ultrashort peptide nanoparticles (USPNs)}

The central aequeous peptide (using peptides LD6, LK6 and IK3) stream was hydrodynamically focused between the side streams containing solvents (methanol, ethanol or HFIP). To Preparation of ultrashort peptide nanoparticles (USPNs) vary the degree of focusing, the flow rate ratio can easily be adjusted by a pumping system (MFCS-FLEX pumping system, Fluigen, France) that incorporates pressurized reservoirs and mass flow controllers in a software-controlled closed loop. For curcumin encapsulation, LK6-biotin was dissolved in pure water at the concentration of $1 \mathrm{mg} / \mathrm{mL}$ and this solution was used for the central stream. Curcumin was dissolved in ethanol and then diluted to $1 \mathrm{mg} / \mathrm{mL}$ in $50 \%$ ethanol and the solution served as side streams.

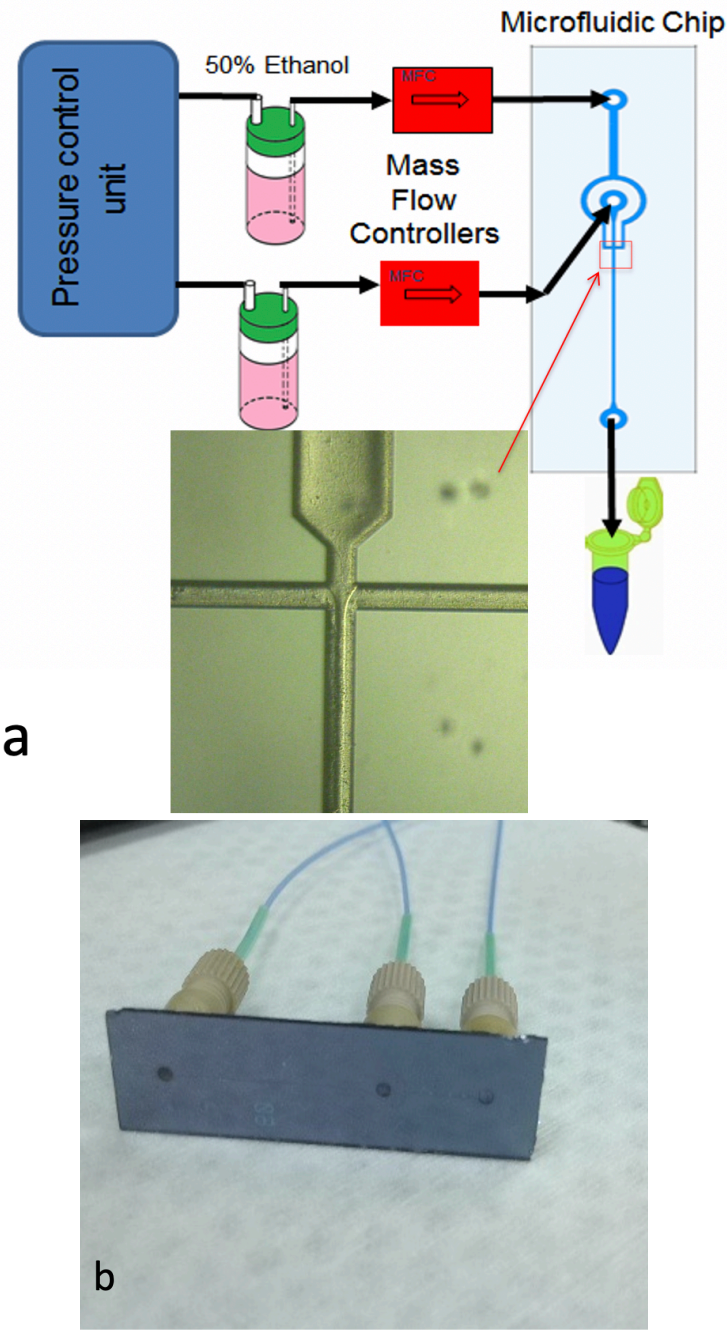

Fig. 1. a) Detailed layout of the microfluidic setup used in this work; b) image with the microfluidic chip with the mounted Nanoport connectors

\section{Characterization of USP nanoparticles}

The size and polydispersity (PDI) of USP nanoparticles were measured by dynamic light scattering (DLS) using a Zetasizer model ZS-90 (Malvern, UK).The morphology of the nanoparticles was visualized by a JSM-7400F field emission scanning electronic microscopy (FE-SEM, JEOL, Tokyo, Japan). The stability of these nanoparticles was monitored by DLS over a period of one week.

\section{E. Cellular uptake of Curcumin-encapsulated USP nanoparticles}

HeLa cells were cultured in Dulbecco's modified Eagle's medium (DMEM) containing high concentration of glucose, supplemented with $10 \%$ fetal bovine serum (FBS, Life Technologies) and $1 \%$ penicillin/streptomycin solution (Gibco®, Life Technologies). Cells were seeded onto four-well chamber slides (SPL, Korea) at a density of $2 \times 10^{4}$ cells/well and cultured overnight. Before adding the nanoparticles, cells were pre-incubated with Opti-MEM (Gibco®, Life technologies) at $37^{\circ} \mathrm{C}$ for $30 \mathrm{~min}$. Curcumin-encapsulated USP 
nanoparticles were first dispersed in Opti-MEM at a peptide concentration of $0.1 \mathrm{mg} / \mathrm{mL}$. These nanoparticles were then incubated with the cells. After $1 \mathrm{~h}$ of incubation, cell medium was removed and cells were fixed with $3.7 \%$ formaldehyde. To visualize peptides, DyLight ${ }^{\mathrm{TM}}$ 488-conjugated NeutrAvidin ${ }^{\mathrm{TM}}$ (Pierce) was added to the cells. The cells were also stained with 4,6-diamidino-2-phenylindole (DAPI, Sigma-Aldrich). The prepared chamber slides were examined by confocal laser scanning microscopy (CLSM, Zeiss LSM 510 META).

\section{F. In Vitro Release of Curcumin from USP Nanoparticles}

Curcumin-encapsulated USP nanoparticles $(0.2 \mathrm{~mL})$, enclosed in Slide-A-Lyzer ${ }^{\mathrm{TM}}$ dialysis cassettes (molecular weight cut-off $(\mathrm{MWCO})=3500)$, were incubated in $100 \mathrm{ml}$ PBS (pH 7.4) at $37^{\circ} \mathrm{C}$ under mild agitation in a water bath. At predetermined time intervals, $1 \mathrm{~mL}$ samples withdrawn from the incubation medium and analyzed for curcumin uptake with fluorescence spectroscopy (Horiba, Japan). Fluorescence intensity of known curcumin concentrations $(0,5,10,20,3040$ $\mathrm{ng} / \mathrm{mL}$ ) was used to establish a standard calibration curve.

\section{Results AND Discussions}

Initially, Ac-LIVAGK-NH 2 peptide ( $\mathrm{LK}_{6}$, American Peptide Company, peptide purity $=98.8 \%$, peptide content $=67.7 \%$ ) was chosen as the model peptide. The hydrodynamic focusing method for the processing of USPNs by employing a microfluidic device has been previously described [22,42]. It can be seen that the central stream is strongly focused by two side streams (Fig. 1a). In order to achieve uniform sized USPNs, the concentration on the central stream $\left(1 \mathrm{mg} / \mathrm{mL} \mathrm{LK}_{6}\right.$ peptide solution) was kept constant while for the side streams different types of solvents were tested. Several chemical reagents were chosen, including methanol, 50/50 ethanol/water and hexafluoroisopropanol (HFIP). The hydrodynamic diameter of USPNs and the size distribution -polydispersity index (PDI) were measured using DLS, (Malvern, UK). Fig. 2 shows that among three different solvents, $50 / 50$ ethanol/water solvent mixture gave nanoparticles with a single peak. Therefore, we focused for further experiments on the ethanolwater mixture as the solvent for the side stream.

Another important aspect is the potential of fine-tuning of the hydrodynamic diameter of USPNs. In this direction, the influence of the concentration of the solvent and the concentration of the peptide solution was studied. Fig. 3a and 3b show the effect of ethanol concentration of the hydrodynamic diameter distribution of USPNs. First, the concentration of $\mathrm{LK}_{6}(1 \mathrm{mg} / \mathrm{mL})$ in the central stream was kept constant and different ethanol concentrations (from $10 \mathrm{vol} \%$ up to $80 \mathrm{vol} \%$ ) in the side streams were tested. It was found that the optimal value, in terms of USPNs size and distribution, of the ethanol concentration is between 50 to $60 \%(\mathrm{vol})$. To investigate the effect of peptide concentration on the particle size and size distribution of USPNs (Fig. 3c and 3d), the ethanol concentration in the side stream was kept constant at $50 \mathrm{vol} \%$ while the peptide concentration $\left(\mathrm{LK}_{6}\right)$ was varied from 10 $\mu \mathrm{g} / \mathrm{mL}$ to $2 \mathrm{mg} / \mathrm{mL}$. It was found that the size of the nanoparticles became smaller as the concentration of the peptide decreased. At the same time, we still achieved uniformsized particles $(\mathrm{PDI}<0.2)$.
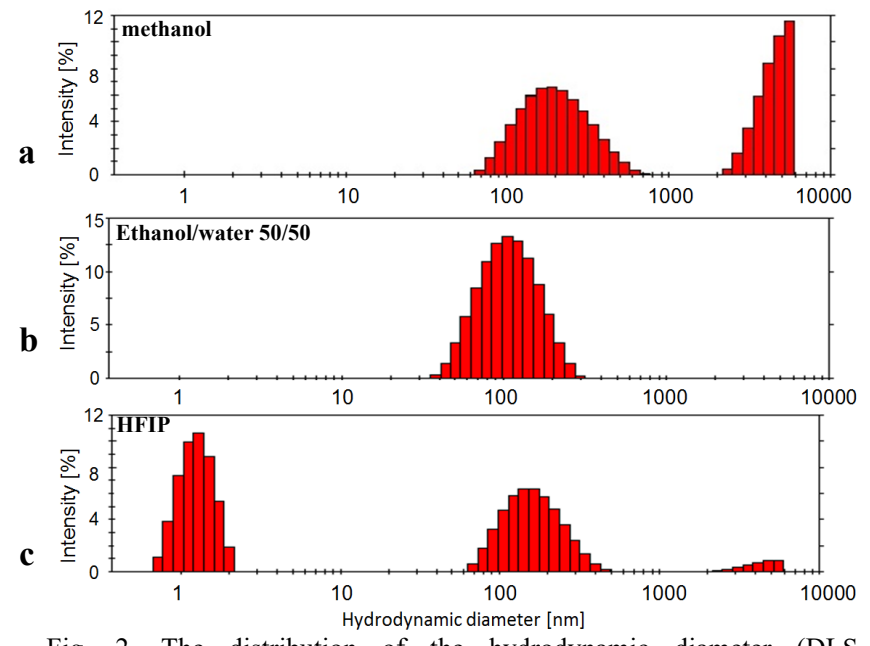

Fig. 2. The distribution of the hydrodynamic diameter (DLS measurement): (a) methanol; (b) 50/50 ethanol/water; (c) 1, 1, 1, 3, 3, 3hexafluoro-2-propanol (HFIP)

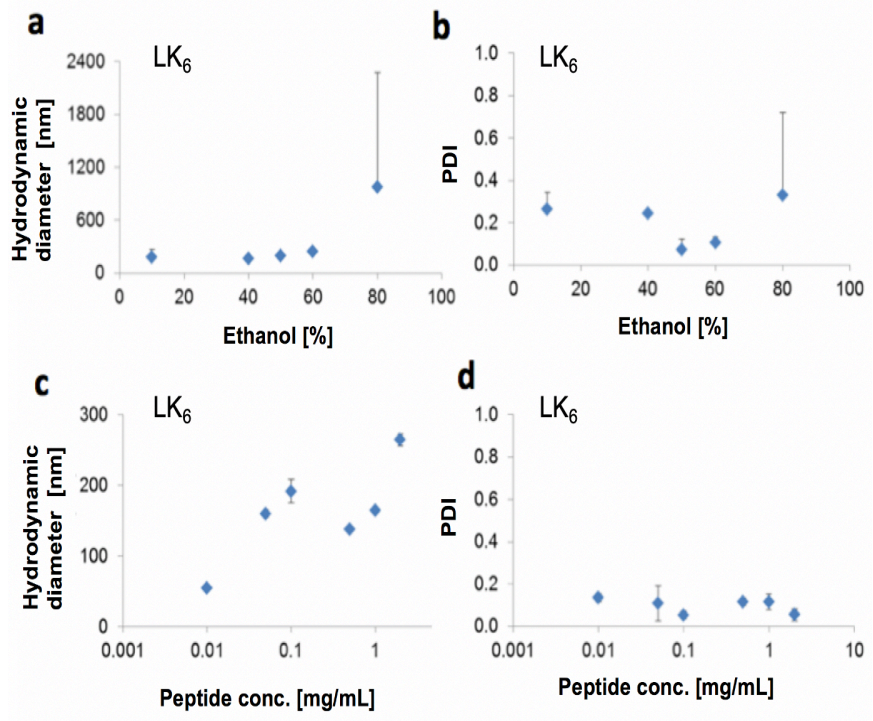

Fig. 3. Effect of ethanol and peptide $\left(\mathrm{LK}_{6}\right)$ concentration on the particle size and size distribution of USPNs. The size distribution was determined by using DLS. Effect of ethanol concentration on the (a) size and (b) polydispersity of USPNs. Effect of peptide concentration on the (c) size and (d) polydispersity of USPNs.

Furthermore, the effect of peptide composition and chain length on the formation of USPNs was investigated. Three different peptides were chosen: Two hexamer peptides $\mathrm{LK}_{6}$ and $\mathrm{LD}_{6}$ and a trimer peptide $\mathrm{IK}_{3}$. All these three peptides, $\mathrm{LK}_{6}, \mathrm{LD}_{6}$ and $\mathrm{IK}_{3}$, belong to the same class of ultrashort self-assembling peptides (33). In Fig. 4, both scanning electron microscopy (SEM) images and DLS measurements showed uniform sized particles. Different to the spherical morphology of the peptide $\mathrm{LK}_{6}$ and $\mathrm{LD}_{6}$ particles, peptide $\mathrm{IK}_{3}$ particles showed an anisotropic particle morphology. It is another signature that peptides were first assembled into nanofibers. [33] 

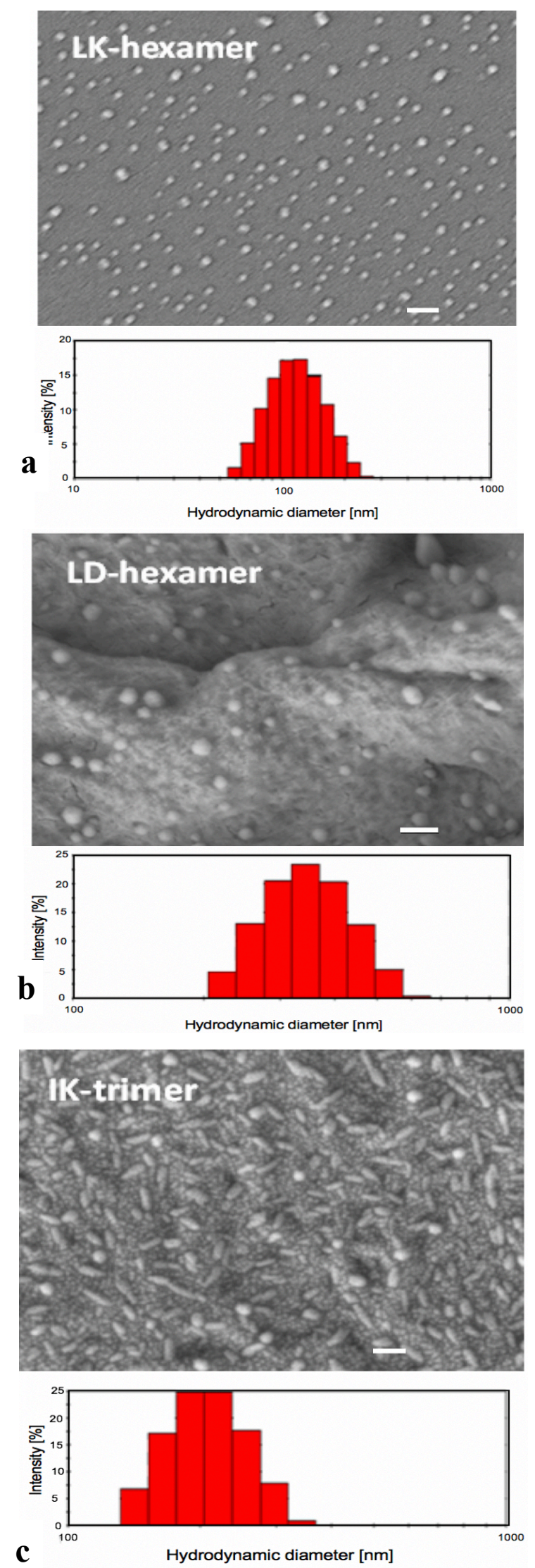

Fig. 4. Comparison of particle size and size distribution of USPNs (SEM pictures +DLS distriburion) prepared from three different ultrashort peptides (a) $\mathrm{LK}_{6}$, (b) $\mathrm{LD}_{6}$, and (c) $\mathrm{IK}_{3}$. Scale bar $=200 \mathrm{~nm}$.
The influence of fluid velocity in the microfluidic channel on the hydrodynamic diameter of the USPN was also investigated. The flow rate ratio peptide $\left(\mathrm{LK}_{6}\right) /$ ethanol was maintained constant while the total flow rate was increased. The results are summarized in Table 1, and it can be noticed that no relevant variations were observed Moreover, we tried to vary the flow rate ratio by keeping the peptide flow rate constant at $3 \mu \mathrm{L} / \mathrm{min}$ and changing the flow rate of the solvent (values of $12 \mu \mathrm{L} / \mathrm{min}$, $15 \mu \mathrm{L} / \mathrm{min}, 18 \mu \mathrm{L} / \mathrm{min}, 21 \mu \mathrm{L} / \mathrm{min}$ and $24 \mu \mathrm{L} / \mathrm{min}$ ). The results are summarized in Table 2 and show a relatively constant value of the hydrodynamic diameter of the nanoparticle with an acceptable value of the polydispersity.

TABLE 1

THE VARIATION OF THE HYDRODYNAMIC DIAMETER OF THE PEPTIDE $\left(\mathrm{LK}_{6}\right)$ NANOPARTICLES WITH THE TOTAL FLOW RATE (VELOCITY OF THE FLUID) AT CONSTANT FLOW RATE RATIO

\begin{tabular}{ccc}
\hline \hline $\begin{array}{c}\text { Flow rate ratio } \\
\text { (peptide/ethanol) }\end{array}$ & $\begin{array}{c}\text { Hydrodynamic } \\
\text { diameter }\end{array}$ & PDI \\
\hline $3: 12$ & $162.9 \pm 0.98$ & 0.016 \\
$4: 16$ & $166.7 \pm 2.01$ & 0.034 \\
$5: 20$ & $166.2 \pm 1.21$ & 0.021 \\
\hline \hline
\end{tabular}

TABLE II

THE VARIATION OF THE HYDRODYNAMIC DIAMETER OF THE USPN (LK 6 ) WITH THE TOTAL FLOW RATE RATIO

\begin{tabular}{ccc}
\hline \hline $\begin{array}{c}\text { Flow rate ratio } \\
\text { (peptide/ethanol) }\end{array}$ & $\begin{array}{c}\text { Hydrodynamic } \\
\text { diameter }\end{array}$ & PDI \\
\hline $3: 12$ & $162.9 \pm 0.98$ & 0.016 \\
$3: 15$ & $163.7 \pm 1.12$ & 0.034 \\
$3: 18$ & $164.1 \pm 1.21$ & 0.021 \\
$3: 21$ & $155.4 \pm 0.61$ & 0.020 \\
$3: 24$ & $155.5 \pm 2,56$ & 0.023 \\
\hline \hline
\end{tabular}

To explore the utility of USPNs as drug carriers, curcumin was encapsulated into USPNs using the microfluidic flow-focusing method. Curcumin-loaded USPNs were incubated with HeLa cells and the cellular uptake of USPNs was visualized by confocal laser scanning microscopy (CLSM). After $1 \mathrm{~h}$ incubation, the cells were fixed with $3.7 \%$ formaldehyde and afterwards the biotin-labelled peptides were stained with NeutrAvidin Dylight ${ }^{\circledR}$ 488. Fluorescence micrographs (Fig. 5) clearly demonstrated the cellular uptake of curcumincontaining peptide nanoparticles by HeLa cells. It can also be seen that the curcumin-containing peptide nanoparticles were present in the cytoplasm (Fig. 5). As a control, we also tested the uptake of curcumin as a free drug (data not shown). Curcumin was found in the cell nucleus but not in the cytoplasm (the mechanism for nucleolar homing of curcumin can be found in [44]).These results clearly show the potential of using USPNs as drug carriers. Curcumin is a promising drug candidate for the treatment of neurodegenerative diseases such as Parkinson's and Alzheimer's diseases due to its neuroprotective effects and ability to cross the blood-brain barrier.[45] However, curcumin suffers from low half-life in the blood stream (5-10 minutes). Therefore, it is essential to use the 
concept of "controlled release" to deliver curcumin in a sustainable way. Hence, curcumin-containing USPNs may be used to repair damaged brain tissue.

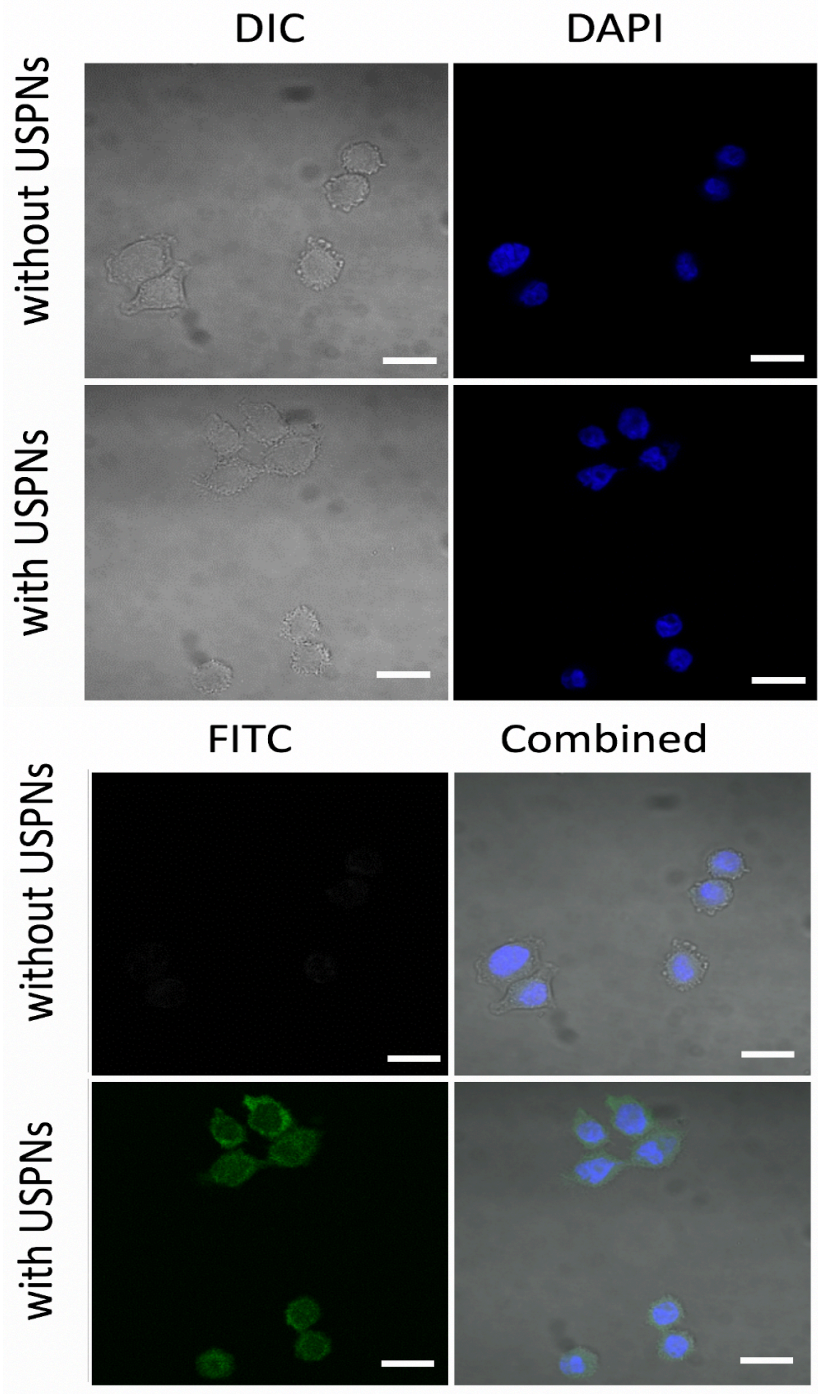

Fig. 5. Cellular uptake of curcumin-loaded $\mathrm{LK}_{6}$-biotin nanoparticles. Upper panel: HeLa cells exposed to normal medium without USPNs (control); Lower panel: HeLa cells exposed to curcumin-loaded $\mathrm{LK}_{6-}$ biotin nanoparticles $\mathrm{b}$ containing medium. Peptides in HeLa cells displayed green color (fluorescence); cell nucleus displayed blue color (DAPI). Scale bars: $20 \mu \mathrm{m}$

The in vitro release of curcumin from the USP nanoparticles was further studied. Curcumin-encapsulated USP nanoparticles $(0.2 \mathrm{~mL})$, enclosed in Slide-A-LyzerTM dialysis cassettes (molecular weight cut-off $(\mathrm{MWCO})=3500$ ), were incubated in $100 \mathrm{ml} \mathrm{PBS} \mathrm{(pH} \mathrm{7.4)} \mathrm{at} 37^{\circ} \mathrm{C}$ under mild agitation in a water bath. At predetermined time intervals, $1 \mathrm{~mL}$ samples were withdrawn from the incubation medium and analyzed for curcumin by fluorescence spectroscopy (Horiba, Japan). Fluorescence intensity of known curcumin concentrations ( 0 to $40 \mathrm{ng} / \mathrm{mL}$ ) was used to establish a standard calibration curve. We have monitored the in vitro curcumin release from USPNs as shown in Fig. 6. Most of the drug was released from the USPNs after $24 \mathrm{~h}$ incubation (much longer time than its halflife in the blood stream).

We found that even after six days, more than $30 \%$ of the drug was still trapped inside the USPNs. These results are encouraging, because we expect that the UPSNs will be degraded inside the cells, which will eventually lead to $100 \%$ drug release. We further monitored the particle size and distribution of USPNs over a period of a week and no significant variation (Table 3 ) in the USPNs size and distribution was observed. Although we did not study the stability of USPNs over a longer period of time, however, these results are encouraging to formulate a controlled-release form of curcumin for therapeutic applications in the near future. In a previous work, we demonstrated that USP particles are second harmonic-active and can be visualized by second harmonic generation microscopy.[41] These unique nonlinear optical properties encourage the use of USPNs as potential theranostic agents (imaging and therapeutics).

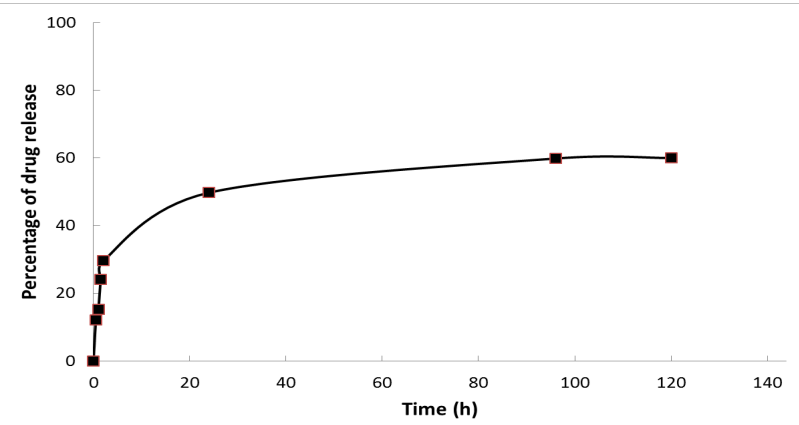

Fig. 6. In vitro release of Curcumin from UPSN

TABLE III

THE HYDRODYNAMIC DIAMETER AND DISTRIBUTION OF CURCUMINLOADED USPNS (AVERAGE \pm STANDARD DEVIATION, $\mathrm{N}=3$ ).

\begin{tabular}{ccc}
\hline \hline Day & $\begin{array}{c}\text { Hydrodynamic } \\
\text { diameter }\end{array}$ & PDI \\
\hline 1 & $68.35 \pm 0.30$ & $0.109 \pm 0.026$ \\
5 & $70.08 \pm 2.73$ & $0.237 \pm 0.043$ \\
6 & $71.15 \pm 3.46$ & $0.285 \pm 0.103$ \\
7 & $74.31 \pm 0.59$ & $0.196 \pm 0.013$ \\
\hline \hline
\end{tabular}

\section{CONCLUSIONS}

In conclusion, we have successfully prepared uniformly sized USPNs using the microfluidic hydrodynamic focusing method and the self-assembly characteristics of USP. These nanoparticles are small in size $(<100 \mathrm{~nm})$ and show good uniformity (PDI $<0.2$ ). The preliminary in vitro curcumin release studies showed that curcumin can be successfully encapsulated into USPNs and slowly released from USPNs. Curcumin-loaded USPNs are stable for at least one week and can be uptaken by mammalian cells. USPNs are also second harmonic-active, which makes them promising candidates as theranostic nanocarriers.

\section{ACKNOWLEDGEMENTS}

$\mathrm{CH}$ wants to acknowledge funding from IBN, A*Star, G.T. acknowledge the funding rom Centre National de la Recherche Scientifique (CNRS, France) through the PICS program 
(project ref. \#6662) and C.I. acknowledge the project NUCLEU IMT MICRO-NANO-SYS +2020".

\section{REFERENCES}

[1] T. MacCulloch, A. Buchberger, and N. Stephanopoulos, "Emerging applications of peptide-oligonucleotide conjugates: bioactive scaffolds, self-assembling systems, and hybrid nanomaterials," Organic \& Biomolecular Chemistry, vol. 17, pp. 1668-1682, 2019.

[2] M. Ni and S. Zhuo, "Applications of self-assembling ultrashort peptides in bionanotechnology," RSC advances, vol. 9, pp. 844-852, 2019.

[3] P. Guo, Y. Wang, and Q. Zhuang, "Highly sensitive and selective biosensor for heparin detection with rhodamine B-labelled peptides as fluorescent bioreceptors," Sensors and Actuators B: Chemical, vol. 299, p. 126873 , 2019.

[4] G.-C. Fan, Z. Li, Y. Lu, L. Ma, H. Zhao, and X. Luo, "Robust photoelectrochemical cytosensor in biological media using antifouling property of zwitterionic peptide," Sensors and Actuators B: Chemical, vol. 299, p. 126996, 2019.

[5] N. Xia, D. Deng, S. Yang, Y. Hao, L. Wang, Y. Liu, et al., "Electrochemical immunosensors with protease as the signal label for the generation of peptide-Cu (II) complexes as the electrocatalysts toward water oxidation," Sensors and Actuators B: Chemical, vol. 291, pp. 113$119,2019$.

[6] K. H. Chan, W. H. Lee, M. Ni, Y. Loo, and C. A. Hauser, "C-Terminal residue of ultrashort peptides impacts on molecular self-assembly, hydrogelation, and interaction with small-molecule drugs," Scientific reports, vol. 8, pp. 1-14, 2018.

[7] J. Xu, N. Liu, C. Hao, Q. Han, Y. Duan, and J. Wu, "A novel fluorescence "on-off-on" peptide-based chemosensor for simultaneous detection of $\mathrm{Cu} 2+, \mathrm{Ag}+$ and S2-," Sensors and Actuators B: Chemical, vol. 280, pp. 129-137, 2019.

[8] T. M. Allen and P. R. Cullis, "Drug delivery systems: entering the mainstream," Science, vol. 303, pp. 1818-1822, 2004.

[9] N. Rapoport, "Physical stimuli-responsive polymeric micelles for anticancer drug delivery," Progress in Polymer Science, vol. 32, pp. 962-990, 2007.

[10]E. Ruoslahti and M. D. Pierschbacher, "New perspectives in cell adhesion: RGD and integrins," Science, vol. 238, pp. 491-497, 1987.

[11] K. H. Chan, B. Xue, R. C. Robinson, and C. A. Hauser, "Systematic moiety variations of ultrashort peptides produce profound effects on selfassembly, nanostructure formation, hydrogelation, and phase transition," Scientific Reports, vol. 7, pp. 1-11, 2017.

[12] M. Ni and S. Zhuo, "A pplications of self-assembling ultrashort peptides in bionanotechnology," RSC Adv, vol. 9, 844-852, 2019.

[13] R. Ischakov, L. Adler-Abramovich, L. Buzhansky, T. Shekhter, and E. Gazit, "Peptide-based hydrogel nanoparticles as effective drug delivery agents," Bioorganic \& medicinal chemistry, vol. 21, pp. 3517-3522, 2013.

[14] K. H. Chan and J. J. J. Tay, "Advancement of Peptide Nanobiotechnology via Emerging Microfluidic Technology," Micromachines, vol. 10, p. 627, 2019.

[15]F. S. Majedi, M. M. Hasani-Sadrabadi, S. H. Emami, M. A. Shokrgozar, J. J. VanDersarl, E. Dashtimoghadam, et al., "Microfluidic assisted selfassembly of chitosan based nanoparticles as drug delivery agents," Lab on a Chip, vol. 13, pp. 204-207, 2013.

[16]S. Amrani and M. Tabrizian, "Characterization of nanoscale loaded liposomes produced by 2D hydrodynamic flow focusing," ACS Biomaterials Science \& Engineering, vol. 4, pp. 502-513, 2018.

[17] M. Michelon, D. R. B. Oliveira, G. de Figueiredo Furtado, L. G. de la Torre, and R. L. Cunha, "High-throughput continuous production of liposomes using hydrodynamic flow-focusing microfluidic devices," Colloids and surfaces B: biointerfaces, vol. 156, pp. 349-357, 2017.

[18] W.-Z. S. Lin and N. Malmstadt, "Liposome production and concurrent loading of drug simulants by microfluidic hydrodynamic focusing," European Biophysics Journal, vol. 48, pp. 549-558, 2019.

[19] J. Xu, S. Zhang, A. Machado, S. Lecommandoux, O. Sandre, F. Gu, et al., "Controllable microfluidic production of drug-loaded PLGA nanoparticles using partially water-miscible mixed solvent microdroplets as a precursor," Scientific Reports, vol. 7, pp. 1-12, 2017.

[20]M. H. Leung and A. Q. Shen, "Microfluidic assisted nanoprecipitation of PLGA nanoparticles for curcumin delivery to leukemia jurkat cells," Langmuir, vol. 34, pp. 3961-3970, 2018.
[21] M. Rhee, P. M. Valencia, M. I. Rodriguez, R. Langer, O. C. Farokhzad, and R. Karnik, "Synthesis of size-tunable polymeric nanoparticles enabled by 3D hydrodynamic flow focusing in single-layer microchannels," Advanced Materials, vol. 23, pp. H79-H83, 2011.

[22]C. Iliescu, C. Mărculescu, S. Venkataraman, B. Languille, H. Yu, and G. Tresset, "On-chip controlled surfactant-DNA coil-globule transition by rapid solvent exchange using hydrodynamic flow focusing," Langmuir, vol. 30, pp. 13125-13136, 2014.

[23]C. Iliescu and G. Tresset, "Microfluidics-driven strategy for size-controlled DNA compaction by slow diffusion through water stream," Chemistry of Materials, vol. 27, pp. 8193-8197, 2015.

[24]Y. Wu, L. Li, Y. Mao, and L. J. Lee, "Static micromixer-coaxial electrospray synthesis of theranostic lipoplexes," ACS nano, vol. 6, pp. 2245-2252, 2012.

[25]M. Ni, G. Tresset, and C. Iliescu, "Self-assembled polysulfone nanoparticles using microfluidic chip," Sensors and Actuators B: Chemical, vol. 252, pp. 458-462, 2017.

[26]Y. Liu, Y. Lu, and G. Luo, "Modified nanoprecipitation method for polysulfone nanoparticles preparation," Soft matter, vol. 10, pp. 34143420, 2014.

[27]R. Karnik, F. Gu, P. Basto, C. Cannizzaro, L. Dean, W. Kyei-Manu, et al., "Microfluidic platform for controlled synthesis of polymeric nanoparticles," Nano letters, vol. 8, pp. 2906-2912, 2008.

[28]D. Liu, H. Zhang, F. Fontana, J. T. Hirvonen, and H. A. Santos, "Current developments and applications of microfluidic technology toward clinical translation of nanomedicines," Advanced drug delivery reviews, vol. 128, pp. 54-83, 2018.

[29]D. Liu, H. Zhang, F. Fontana, J. T. Hirvonen, and H. A. Santos, "Microfluidic-assisted fabrication of carriers for controlled drug delivery," Lab on a Chip, vol. 17, pp. 1856-1883, 2017.

[30] I. Lignos, R. Maceiczyk, and A. J. deMello, "Microfluidic technology: Uncovering the mechanisms of nanocrystal nucleation and growth," Accounts of chemical research, vol. 50, pp. 1248-1257, 2017.

[31]P. M. Valencia, O. C. Farokhzad, R. Karnik, and R. Langer, "Microfluidic technologies for accelerating the clinical translation of nanoparticles," Nature nanotechnology, vol. 7, p. 623, 2012.

[32] M. Lu, A. Ozcelik, C. L. Grigsby, Y. Zhao, F. Guo, K. W. Leong, et al., "Microfluidic hydrodynamic focusing for synthesis of nanomaterials," Nano Today, vol. 11, pp. 778-792, 2016.

[33]C. A. E. Hauser, R. Deng, A. Mishra, Y. Loo, U. Khoe, F. Zhuang, et al., "Natural tri- to hexapeptides self-assemble in water to amyloid $\beta$-type fiber aggregates by unexpected $\alpha$-helical intermediate structures," Proceedings of the National Academy of Sciences of the United States of America, vol. 108, pp. 1361-1366, 2011.

[34]Y. Loo, A. Lakshmanan, M. Ni, L. L. Toh, S. Wang, and C. A. Hauser, "Peptide bioink: self-assembling nanofibrous scaffolds for threedimensional organotypic cultures," Nano letters, vol. 15, pp. 6919-6925, 2015.

[35] C. L. Hedegaard, E. C. Collin, C. Redondo-Gómez, L. T. Nguyen, K. W. Ng, A. A. Castrejón-Pita, et al., "Hydrodynamically Guided Hierarchical Self-Assembly of Peptide-Protein Bioinks," Advanced Functional Materials, vol. 28, p. 1703716, 2018.

[36] H. P. Felgueiras and M. T. P. Amorim, "Functionalization of electrospun polymeric wound dressings with antimicrobial peptides," Colloids and Surfaces B: Biointerfaces, vol. 156, pp. 133-148, 2017.

[37] Y. Loo, Y.-C. Wong, E. Z. Cai, C.-H. Ang, A. Raju, A. Lakshmanan, et al., "Ultrashort peptide nanofibrous hydrogels for the acceleration of healing of burn wounds," Biomaterials, vol. 35, pp. 4805-4814, 2014.

[38]K. H. Chan, S. Zhuo, and M. Ni, "Priming the surface of orthopedic implants for osteoblast attachment in bone tissue engineering," International Journal of Medical Sciences, vol. 12, pp. 701-707, 2015.

[39]M. R. Reithofer, K.-H. Chan, A. Lakshmanan, D. H. Lam, A. Mishra, B. Gopalan, et al., "Ligation of anti-cancer drugs to self-assembling ultrashort peptides by click chemistry for localized therapy," Chemical science, vol. 5, pp. 625-630, 2014.

[40]B. Ma, F. Niu, X. Qu, W. He, C. Feng, S. Wang, et al., "A tetrameric protein scaffold as a nano-carrier of antitumor peptides for cancer therapy," Biomaterials, vol. 204, pp. 1-12, 2019.

[41] M. Ni, S. Zhuo, C. Iliescu, P. T. So, J. S. Mehta, H. Yu, et al., "Selfassembling amyloid-like peptides as exogenous second harmonic probes for bioimaging applications," Journal of biophotonics, p. e201900065, 2019.

[42] G. Tresset, C. Marculescu, A. Salonen, M. Ni, and C. Iliescu, "Fine control over the size of surfactant-polyelectrolyte nanoparticles by hydrodynamic flow focusing," Analytical chemistry, vol. 85, pp. 5850-5856, 2013 
[43]C. Iliescu, H. Taylor, M. Avram, J. Miao, and S. Franssila, "A practical guide for the fabrication of microfluidic devices using glass and silicon," Biomicrofluidics, vol. 6, pp. 16505-1650516, Mar 2012.

[44] M. Ghosh, and R. O. Ryan, Curcumin homing to the nucleolus: mechanism for initiation of an apoptotic program. The Journal of nutritional biochemistry, 25(11), pp.1117-1123. 2014.

[45]Y.-M. Tsai, C.-F. Chien, L.-C. Lin, and T.-H. Tsai, "Curcumin and its nanoformulation: the kinetics of tissue distribution and blood-brain barrier penetration," International journal of pharmaceutics, vol. 416, pp. 331338,2011

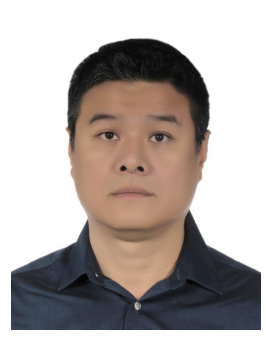

Ming Ni received his Ph.D. in Chemical Engineering from the University of Washington in 2004. He was a research scientist at the Institute of Bioengineering and Nanotechnology, Agency for Science, Technology and Research in Singapore from 2007 to 2016. He was professor and Head of the Biomedical Engineering Department, Yachay Tech University. Currently is project manager with GeneScript, China. His research focuses on biomaterials and nanomaterials. He is also interested in microfluidics, stem cell therapy, bioimaging and drug delivery.

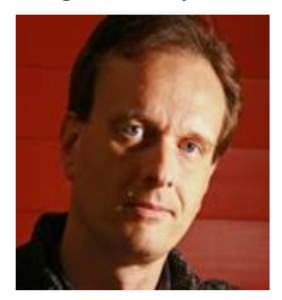

Guillaume Tresset earned his MS degree in electrical engineering in 1998 from Ecole Supérieur d'Electricité and a PhD in physics in 2002 from Aix-Marseille Université in France. He is currently a CNRS research director in the department of physics at the Université Paris-Saclay. His research interests cover self-assembly phenomena in biological matter and microfluidics.

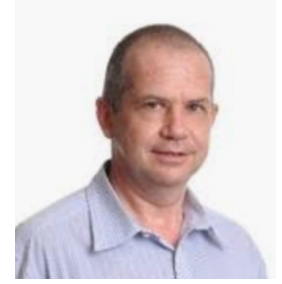

Ciprian Iliescu received his Ph.D. from Polytechnic University of Bucharest in 1999. During his $\mathrm{PhD}$ studies he also worked at Baneasa S.A. (IC's company). He spun off a company with his $\mathrm{PhD}$ work on silicon pressure sensors. He worked as a Post Doc with the Micromachines Center at the Nanyang Technological University, Singapore. Further he joined the Institute of Bioengineering and Nanotechnology and BIGHEART team at National University of Singapore. Currently, he joined IMT Bucharest as senior scientist. His current research interest concerns the development of biomedical devices, microfluidics and bioengineering. $\mathrm{He}$ is member of the Academy of Romanian Scientists.

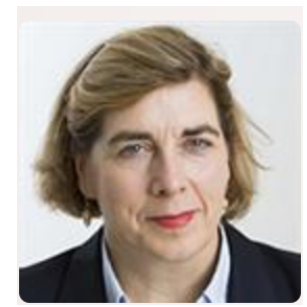

Charlotte A.E. Hauser is professor of bioscience and chair of the Bioengineering program at King Abdullah University of Science and Technology (KAUST), Saudi Arabia. Before joining KAUST, she was a principal investigator at the Institute of Bioengineering and Nanotechnology, A*Star, Singapore, and adjunct professor at Nanyang Technological University, Singapore. She did her PhD work in molecular biology at the Massachusetts Institute of Technology (MIT) under Prof. Alexander Rich. In 1997, she founded Octagene as a joint venture in Munich/Martinsried, Germany, where the first fourth generation human recombinant coagulation factor VIII (hFVIII) was developed. The factor with the trade name NUWIQ ${ }^{\circledR}$ is since 2014 on the market. Professor Hauser is a Fellow of the American Institute for Medical and Biological Engineers (AIMBE) and a Fellow of the US National Academy of Inventors (NAI). In 2016, she started the KAUST spin-off company PepPrint. Professor Hauser's research interests align at the interfaces between chemistry, biomedicine, bioengineering and nanotechnology. Focus is on the development of platform technologies, using smart nanomaterials for regenerative, biomedical and environmental. Furthermore, Professor Hauser is interested in $3 \mathrm{D}$ bioprinting, using supramolecular organotypic constructs to fabricate high-throughput platforms for drug screening, pathogen detection and other diagnostic purposes. Synthetic biology approaches are explored for the generation of functional biomaterial. 